\title{
A CT calibration method based on the polybinary tissue model for radiotherapy treatment planning
}

\author{
Nobuyuki Kanematsu $\dagger$, Naruhiro Matsufuji, Ryosuke \\ Kohno, Shinichi Minohara and Tatsuaki Kanai \\ Research Center for Charged Particle Therapy, National Institute of \\ Radiological Sciences, 4-9-1 Anagawa, Inage-Ku, Chiba 263-8555, Japan
}

\begin{abstract}
A method to establish the relationship between CT number and effective density for therapeutic radiations is proposed. We approximated body tissues to mixtures of muscle, air, fat, and bone. Consequently, the relationship can be calibrated only with a CT scan of their substitutes, for which we chose water, air, ethanol, and potassium phosphate solution, respectively. With simple and specific corrections for non-equivalencies of the substitutes, the calibration accuracy of $1 \%$ will be achieved. We tested the calibration method with some biological materials to verify that the proposed method would offer accuracy, simplicity, and specificity required for a standard in radiotherapy treatment planning, in particular, with heavy charged particles.
\end{abstract}

PACS numbers: 87.53Tf, 87.53Xd, 87.59Fm

Submitted to: Phys. Med. Biol.

\section{Introduction}

Dose calculations in radiotherapy treatment planning are usually based on depthdose curves measured in water with corrections for longitudinal heterogeneity by the effective depth calculation, which is a linear integration of the effective density relative to water along the beam path. The effective density of a material depends not only on its chemical compositions and physical density but also on the radiation of interest. For therapeutic $\mathrm{x}$ rays generated by megavoltage electron accelerators, where Compton scattering dominates the interactions with tissues, the effective density is approximated to the relative electron density. For charged particle beams, the effective density should be by definition the relative stopping power.

Currently, x-ray computed tomography (CT) is widely used in radiotherapy treatment planning to obtain the volumetric distribution of so-called CT number in a patient. The $\mathrm{CT}$ number is directly related to the linear attenuation coefficient for the $\mathrm{x}$ ray and is usually calibrated to 0 for water and to -1000 for air. Since commercially available $\mathrm{CT}$ scanners use kilovoltage $\mathrm{x}$ rays, where the photoelectric effect and the coherent scattering play substantial roles, the x-ray attenuation depends not only on the electron density but also on atomic composition of the material. In addition, the

$\dagger$ E-mail address: nkanemat@nirs.go.jp 
CT number also depends on photon energy spectrum, geometrical configuration of the phantom system, detector sensitivity, and possibly reconstruction algorithm.

Therefore, the relationship between CT number and effective density for body tissues should be calibrated specifically for each scanning condition of each CT system. IEC (1994) specified various monthly and semiannual constancy tests for CT scanners, which should naturally be extended to the relationship for quality assurance of radiotherapy. Constantinou et al (1992) estimated the typical precision of CT systems to $1 \%-2 \%$ in attenuation, based on the observed position dependence in a phantom and day-to-day reproducibility. In addition, changes of the effective depth of a tumor can be even larger, caused by body deformations, over a treatment course of typically weeks long. Therefore, in the current treatment procedure relying on the past patient images, calibration uncertainties of order of $1 \%$ in effective depth may be tolerated.

The relationship determined by a measured correlation between CT numbers and the effective densities for various body tissues, which are approximately substituted by available artificial materials in the tissue-substitute calibration. Chen et al (1977) used solutions of water and potassium phosphate $\left(\mathrm{K}_{2} \mathrm{HPO}_{4}\right)$ for bones, ethanol and water mixtures for adipose tissues, and cork for lung. Constantinou et al (1992) used a commercial phantom system with various solid materials of known atomic compositions and deduced a relationship relating the CT number to the electron density for photon radiotherapy. Similarly, Jäkel et al (2001) used solid tissue substitutes for heavy-ion radiotherapy. However, those artificial materials might not sufficiently be equivalent to the real tissues for charged particle radiotherapy, where very accurate depth control is required. Schneider et al (1996) and Matsufuji et al (1998) estimated the inaccuracy with the tissue-substitute calibration to a few percent in effective density. Rietzel et al (2000) used real animal tissues to resolve the problem, though measurements of various raw tissues with $\mathrm{CT}$ and therapeutic beams in the identical condition may be technically difficult in practice.

ICRU (1989 and 1992) reported comprehensive material information on the standard body tissues, which enabled the quantitative understanding of interactions of radiations with body tissues in conjunction with knowledge on interactions of radiations with matter (Jackson and Hawkes 1981 and ICRU 1984 and 1993). Schneider et al (1996) took such a theoretical approach, the stoichiometric calibration, for radiotherapy treatment planning. They measured CT numbers for artificial tissue substitutes to determine the characteristic parameters for their CT scanner and theoretically predicted the CT numbers for the various body tissues, which were then related to the effective densities. Schaffner and Pedroni (1998) verified the validity of this method for proton radiotherapy in more detail. The stoichiometric calibration will give various body tissue responses from a limited number of measurements for the model parameter determination, for which inaccurate tissue substitutes may be used as long as their atomic compositions are precisely known. However, the involved theoretical calculations may be too complicated to practice without complete understanding of the underlying physics.

In recent years, facilities for heavy charged particle radiotherapy have been increasing worldwide and it is becoming of more importance to establish the quality standard for treatments of this category, which has to be equally achievable by the relevant facilities. We are proposing here a new calibration method that offers the accuracy of the stoichiometric calibration and the simplicity of the tissue-substitute calibration, motivated by the principle of balancing quality and cost for quality 
assurance of radiotherapy (Kutcher et al 1993).

\section{Materials and methods}

\subsection{The stoichiometric calibration}

We based the theoretical model on the stoichiometric calibration by Schneider et al (1996), which is briefly reviewed here. The formalism for photon attenuation by Rutherford et al (1976) gives the linear attenuation coefficient of a material relative to that of water $\mu$ by

$$
\mu=\rho_{\mathrm{e}}\left(K^{\mathrm{ph}} \tilde{Z}^{m-1}+K^{\mathrm{coh}} \hat{Z}^{n-1}+\sigma^{\mathrm{KN}}\right)
$$

where $K^{\mathrm{ph}}, K^{\mathrm{coh}}$, and $\sigma^{\mathrm{KN}}$ are the coefficients for photoelectric effect, coherent scattering, and Compton scattering, respectively, which depend on the photon energy spectrum of a CT scanner. Relative electron density $\rho_{\mathrm{e}}$, defined by the number of electrons per unit volume of the material divided by that of water, can be calculated from the given physical density and atomic composition of the material. Quantities $\tilde{Z}$ and $\hat{Z}$ are the effective atomic numbers of the material for photoelectric effect and coherent scattering, respectively, and exponents $m$ and $n$ are approximately constant and were optimized to 4.62 and 2.86 , respectively, for attenuation of $60-80-\mathrm{keV}$ photon in oxygen representing a typical CT-scanning condition. The mixture rule then gives the effective atomic numbers by

$$
\begin{aligned}
& \tilde{Z}=\left(\sum_{i} \lambda_{i} Z_{i}^{m-1}\right)^{1 /(m-1)} \\
& \hat{Z}=\left(\sum_{i} \lambda_{i} Z_{i}^{n-1}\right)^{1 /(n-1)}
\end{aligned}
$$

where weight $\lambda_{i}$ is the fraction of electrons attributed to atom $i$ in the compound. With one of the CT scanners at National Institute of Radiological Sciences (NIRS), we measured CT numbers for materials of known compositions and obtained the best fit parameters $K^{\mathrm{ph}}=2.9733 \times 10^{-5}, K^{\mathrm{coh}}=1.0665 \times 10^{-3}$, and $\sigma^{\mathrm{KN}}=0.91476$ after conversions to relative linear attenuation coefficients by

$$
\mu=\frac{H-H_{\text {air }}}{H_{\text {water }}-H_{\text {air }}}
$$

where $H, H_{\text {air }}$, and $H_{\text {water }}$ are the measured CT numbers for the material of interest, air, and water, respectively. The measurements of water and air are intended to minimize common errors in the calibration CT scan, assuming that CT number will be always well calibrated with water and air for planning CT scans.

On the other hand, the effective density for radiotherapy is either simply $\rho_{\mathrm{e}}$ for therapeutic megavoltage photons or the stopping power relative to water $\rho_{\mathrm{S}}$ for charged particles that can be approximated to

$$
\rho_{\mathrm{S}}=\rho_{\mathrm{e}}\left[\ln \left(\frac{2 m_{\mathrm{e}} c^{2}}{I} \frac{\beta^{2}}{1-\beta^{2}}\right)-\beta^{2}\right]\left[\ln \left(\frac{2 m_{\mathrm{e}} c^{2}}{I_{\text {water }}} \frac{\beta^{2}}{1-\beta^{2}}\right)-\beta^{2}\right]^{-1}
$$

where constants $m_{\mathrm{e}}$ and $c$ are the electron mass and the speed of light, respectively, $\beta$ is the velocity of the charged particle relative to $c$, and $I$ and $I_{\text {water }}(=75 \mathrm{eV})$ are the mean excitation energies for the medium and water, respectively (ICRU 1984). 
The change of $\rho_{\mathrm{S}}$ will be only $0.5 \%$ between $\beta=0.4$ and 0.7 even for cortical bone with $I=106 \mathrm{eV}$ (ICRU 1993), which is the worst case, according to (5). Since this $\beta$ range covers kinetic energy per nucleon $E / A=80$ to $370 \mathrm{MeV}$ or residual range $R=$ 6 to $75 \mathrm{~cm}(2$ to $25 \mathrm{~cm}$ ) for proton (carbon), we can safely approximate the effective velocity in body to constant $\beta=0.6(E / A=230 \mathrm{MeV})$ for therapeutic heavy charged particles up to carbon.

The radiological properties for a series of body tissue compositions listed by ICRU (1992) were calculated and plotted in figure 1, which include adipose tissues, bloods, brains, eye lens, a GI tract, hearts, kidneys, livers, lungs, a lymph, muscles, an ovary, a pancreas, placentae, a cartilage, cortical bones, a cranium, femurs, a humerus, a mandible, a red marrow, ribs, sacra, a spongiosa, a vertebral column, an yellow marrow, skins, spleens, a testis, a thyroid, and urinary bladders. The relative linear attenuation coefficients were calculated using (1) with the parameters for the example CT scanner, the electron densities were given by ICRU (1992), and the relative stopping powers were calculated using (5) with $I$ values given by the mixture rule for atomic constituents of compounds by ICRU (1984). The calculated tissue responses in either correlation $\mu-\rho_{\mathrm{e}}$ or $\mu-\rho_{\mathrm{S}}$ fit well to a single polyline consisting of line segments for lung tissues $(\mu \leq 0.6)$, soft tissues $(0.85 \leq \mu \leq 1.1)$, bones $(\mu \geq 1.3)$, and their gaps $(0.6<\mu<0.85$ and $1.1<\mu<1.3)$. Since the $\mu$ ranges for those tissue types hardly depend on individual CT scanners and, in addition, few tissues fall in the gap regions, the divisions of the $\mu$ ranges were determined to reasonably minimize the kinks. The ICRU tissues distribute around the polyline with RMS below 0.007 in relative effective density as shown in figure $1(e)$ or figure $1(f)$, which may be the ideal resolution for the correlation under such assumptions that the polyline structure is a nature of body tissues and that the residuals are dominated by variation among individuals rather than tissue types. The calibration should be a procedure to minimize additional errors to the ideal resolution.

\subsection{The polybinary tissue model}

For a mixture of two components (a binary system), radiological properties $\mu, \rho_{\mathrm{e}}$, and $\rho_{\mathrm{S}}$ should be all linearly related to the mixing ratio and thus also to one another. The polyline structure observed in figure 1 naturally implies that the body tissues are in such binary systems. Since muscle occupies the largest content of body (ICRP 1975) and the ICRU muscles were in the middle of the spot where many tissues concentrated in figure 1, we took muscle for one component of the binary systems and approximated lung tissues, soft tissues, and bones to mixtures of muscle-air, musclefat, and muscle-bone mineral, respectively. Once radiological properties $\mu, \rho_{\mathrm{e}}$, and $\rho_{\mathrm{S}}$ for the body tissue components are evaluated, it will be straightforward to draw the polyline relating between them.

We looked for materials representing those components, which should be available, universal, and reasonably tissue-equivalent. Equations (1) and (5) indicate that quantities $\rho_{\mathrm{e}}, \tilde{Z}, \hat{Z}$, and $I$ determine the relevant radiological properties of materials. Since $\rho_{\mathrm{e}}$ is exactly calculable for materials of known compositions and is simply a multiplicative factor for observables $\mu$ and $\rho_{\mathrm{S}}$, differences of $\rho_{\mathrm{e}}$ can be easily corrected by scaling. Therefore, we evaluated the similarity of the materials only with $\tilde{Z}, \hat{Z}$, and $I$ to choose the optimum substitutes. Figure 2 shows the calculated atomic characteristics of the ICRU body tissues and the chosen substitutes, which are ethanol for adipose tissue, water for muscle, and $40 \%-\mathrm{K}_{2} \mathrm{HPO}_{4}$ water solution (by weight) for 

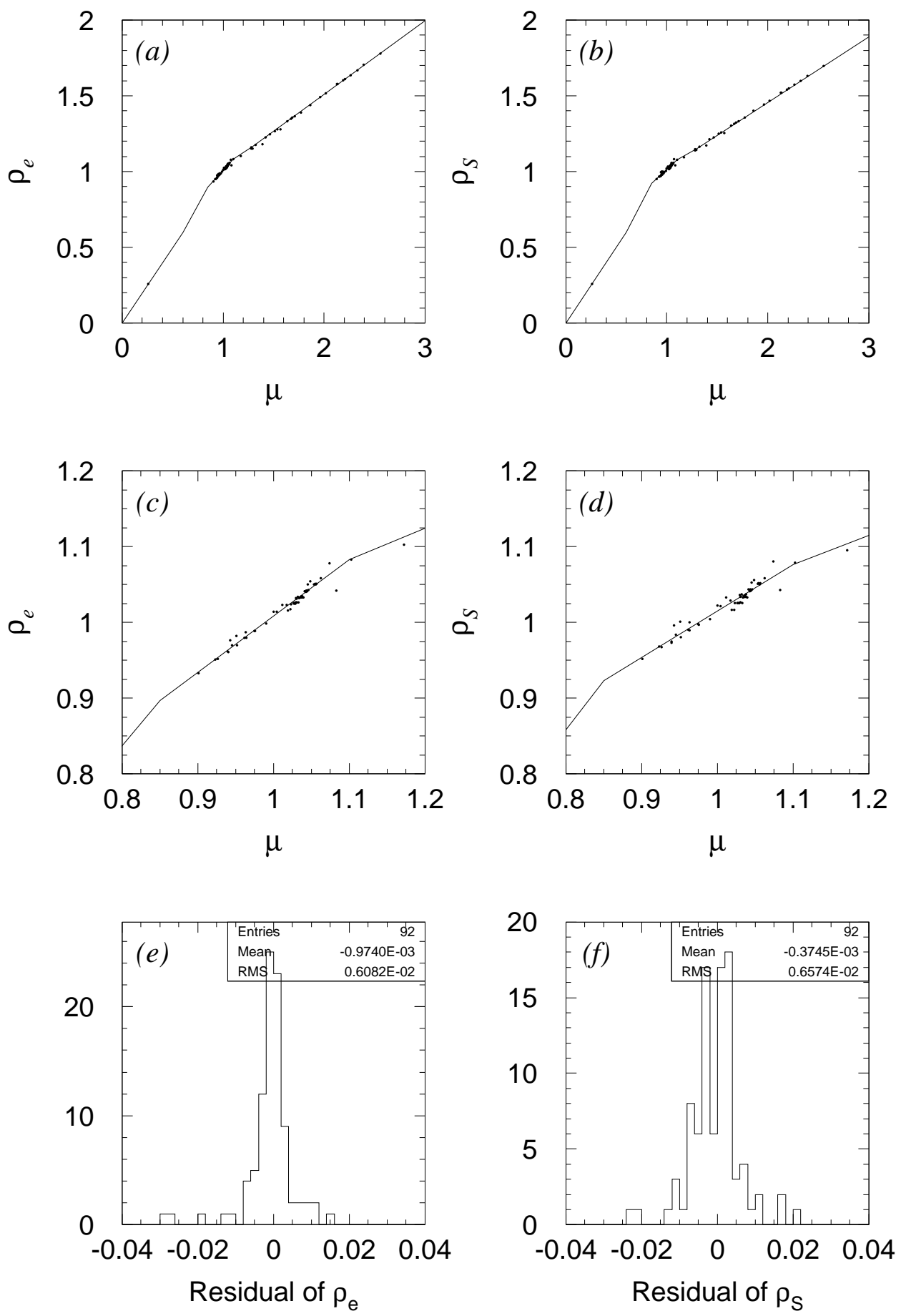

Figure 1. Radiological properties of the body tissue compositions in an example stoichiometric calibration; $(a)$ and $(c)$ the correlation between relative linear attenuation coefficient $\mu$ and relative electron density $\rho_{\mathrm{e}},(b)$ and $(d)$ correlation between $\mu$ and relative stopping power $\rho_{\mathrm{S}}$, and $(e)$ and $(f)$ residual histograms of $\rho_{\mathrm{e}}$ and $\rho_{\mathrm{S}}$, respectively, with respect to the polylines. 
Table 1. Atomic and radiological properties for the tissue substitutes and the corresponding tissue components. A tissue component with relative electron density $\rho_{\mathrm{e} t}$ and relative stopping power $\rho_{\mathrm{S} t}$ will have the same effective atomic number $\tilde{Z}$ as that for the substitute with relative electron density $\rho_{\mathrm{e} s}$.

\begin{tabular}{lllllll}
\hline Substitute & $\tilde{Z}_{s}$ & $\hat{Z}_{s}$ & $\rho_{\mathrm{e} s}$ & Tissue & $\rho_{\mathrm{et}}$ & $\rho_{\mathrm{S} t}$ \\
\hline Ethanol & 6.497 & 5.984 & 0.801 & Adipose & 0.951 & 0.968 \\
Water & 7.522 & 7.115 & 1 & Muscle & 1.029 & 1.032 \\
$40 \%-\mathrm{K}_{2} \mathrm{HPO}_{4}$ & 12.51 & 10.555 & 1.344 & Bone & 1.399 & 1.362 \\
\hline
\end{tabular}

bone. The body tissues showed a strong correlation among $\tilde{Z}, \hat{Z}$, and $I$, with which the adopted substitutes were also consistent. They were in fact superior to the majority of commercial tissue substitutes in terms of atomic similarity to the body tissues. The physical densities relative to water at room temperature were measured to be 0.788 for ethanol and 1.406 for $40 \%-\mathrm{K}_{2} \mathrm{HPO}_{4}$ water solution, which determined the relative electron densities.

Since the $\hat{Z}^{n-1}$ term in equation (1) is generally less important (Schaffner and Pedroni 1998) and the proposed calibration does not rely on the stopping powers of the substitutes, we defined the components of the polybinary tissue model (muscle, adipose tissue, and bone) as the ICRU body tissues corresponding to the substitutes through $\tilde{Z}$, ignoring small differences in $\hat{Z}$ and $I$. Figure 3 shows the correlation between the effective atomic number $\tilde{Z}$ and the effective density, either $\rho_{\mathrm{e}}$ for megavoltage x rays or $\rho_{\mathrm{S}}$ for therapeutic heavy charged particles, for the ICRU body tissues. The $\rho_{\mathrm{e}}$ and $\rho_{\mathrm{S}}$ values of the tissue components were obtained by the linear fits and are shown in table 1 , while equation (1) gives the $\mu$ values of the tissue components by scaling

$$
\mu_{t}=\frac{\rho_{\mathrm{e} t}}{\rho_{\mathrm{es}}} \mu_{s}
$$

where suffices $s$ and $t$ specify a substitute and the corresponding tissue component, namely, ethanol and the adipose tissue, water and the muscle, or $40 \%-\mathrm{K}_{2} \mathrm{HPO}_{4}$ solution and the bone. The polybinary tissue model gives the relationship between relative linear attenuation coefficient $\mu$ and generic effective density $\rho$ for lung tissues, soft tissues, and bones by

$$
\begin{array}{ll}
\rho=\rho_{\mathrm{M}}+\frac{\rho_{\mathrm{M}}}{\mu_{\mathrm{M}}}\left(\mu-\mu_{\mathrm{M}}\right) & (\mu \leq 0.6) \\
\rho=\rho_{\mathrm{M}}+\frac{\rho_{\mathrm{A}}-\rho_{\mathrm{M}}}{\mu_{\mathrm{A}}-\mu_{\mathrm{M}}}\left(\mu-\mu_{\mathrm{M}}\right) & (0.85 \leq \mu \leq 1.1) \\
\rho=\rho_{\mathrm{M}}+\frac{\rho_{\mathrm{B}}-\rho_{\mathrm{M}}}{\mu_{\mathrm{B}}-\mu_{\mathrm{M}}}\left(\mu-\mu_{\mathrm{M}}\right) & (\mu \geq 1.3)
\end{array}
$$

respectively, where $\rho_{\mathrm{A}}, \mu_{\mathrm{A}}, \rho_{\mathrm{M}}, \mu_{\mathrm{M}}, \rho_{\mathrm{B}}$, and $\mu_{\mathrm{B}}$ are the effective densities and the relative linear attenuation coefficients for the adipose tissue, the muscle, and the bone, respectively, given in table 1 . Calculations of equations (7)-(9) at boundaries $\mu=0.6$, $0.85,1.1$, and 1.3 with the values in table 1 converted by (6) give the vertices of the polyline as shown in table 2 , where the relative linear attenuation coefficient for ethanol $\mu_{\mathrm{E}}$ and that for $40 \%-\mathrm{K}_{2} \mathrm{HPO}_{4}$ solution $\mu_{\mathrm{K}}$ are scanning-condition specific parameters to be measured. 

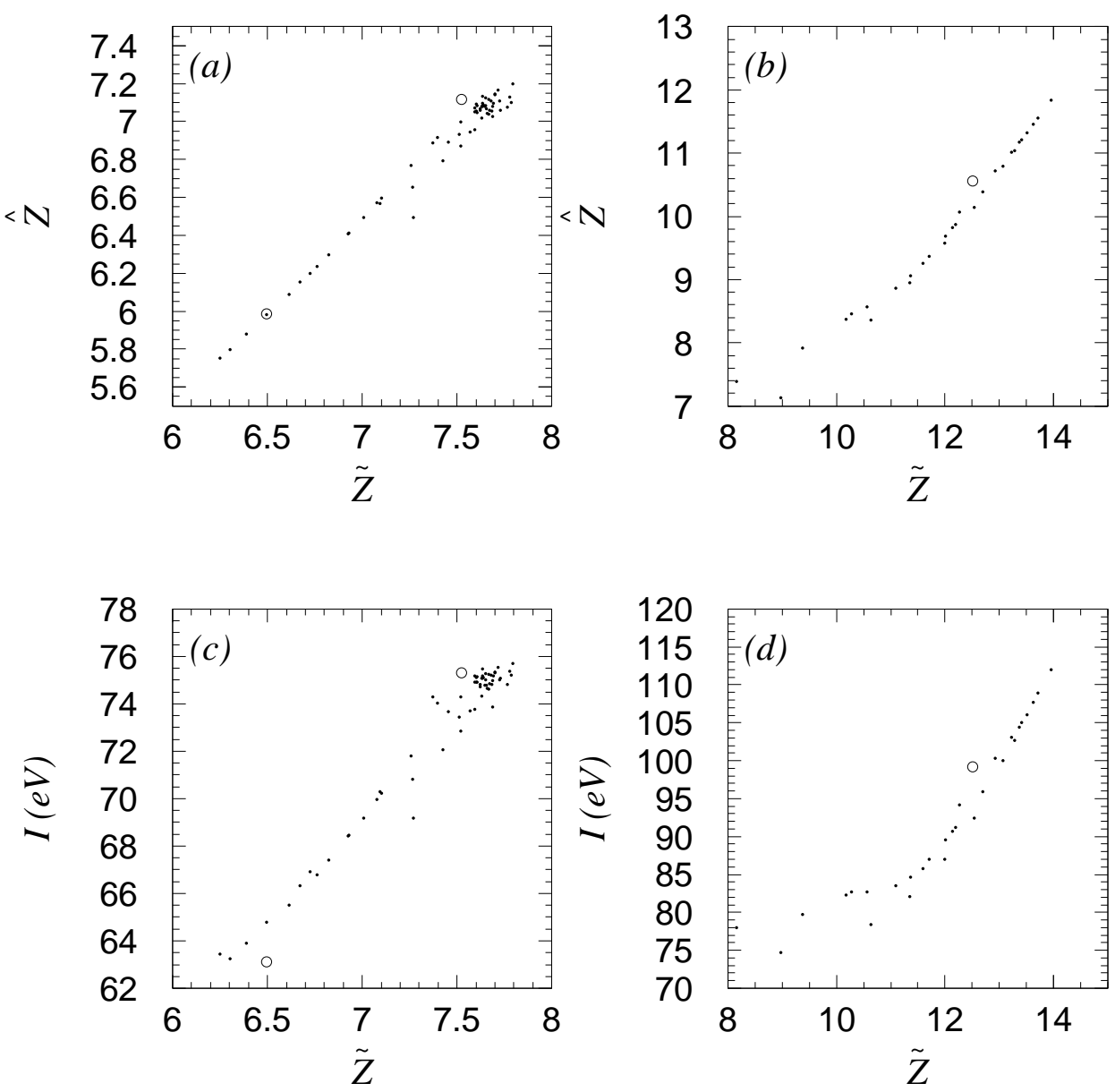

Figure 2. Correlation among the atomic properties of body tissues; (a) $\tilde{Z}-\hat{Z}$ for soft tissues, (b) $\tilde{Z}-\hat{Z}$ for bones, (c) $\tilde{Z}-I$ for soft tissues, and (d) $\tilde{Z}-I$ for bones, where the circles indicate ethanol, water, and $40 \%-\mathrm{K}_{2} \mathrm{HPO}_{4}$ solution.

Table 2. Vertices of the polyline given by the polybinary calibration, where $\mu_{\mathrm{E}}$ and $\mu_{\mathrm{K}}$ are the observed relative linear attenuation coefficients for ethanol and $40 \%-\mathrm{K}_{2} \mathrm{HPO}_{4}$ solution, respectively.

\begin{tabular}{lll}
\hline$\mu$ & $\rho_{\mathrm{e}}$ & $\rho_{\mathrm{S}}$ \\
\hline 0 & 0 & 0 \\
0.6 & 0.600 & 0.602 \\
0.85 & $1.029-0.01176 /\left(0.8667-\mu_{\mathrm{E}}\right)$ & $1.032-0.009649 /\left(0.8667-\mu_{\mathrm{E}}\right)$ \\
1.1 & $1.029+0.004664 /\left(0.8667-\mu_{\mathrm{E}}\right)$ & $1.032+0.003827 /\left(0.8667-\mu_{\mathrm{E}}\right)$ \\
1.3 & $1.029+0.09633 /\left(\mu_{\mathrm{K}}-0.9885\right)$ & $1.032+0.08591 /\left(\mu_{\mathrm{K}}-0.9885\right)$ \\
3 & $1.029+0.7006 /\left(\mu_{\mathrm{K}}-0.9885\right)$ & $1.032+0.6249 /\left(\mu_{\mathrm{K}}-0.9885\right)$ \\
\hline
\end{tabular}



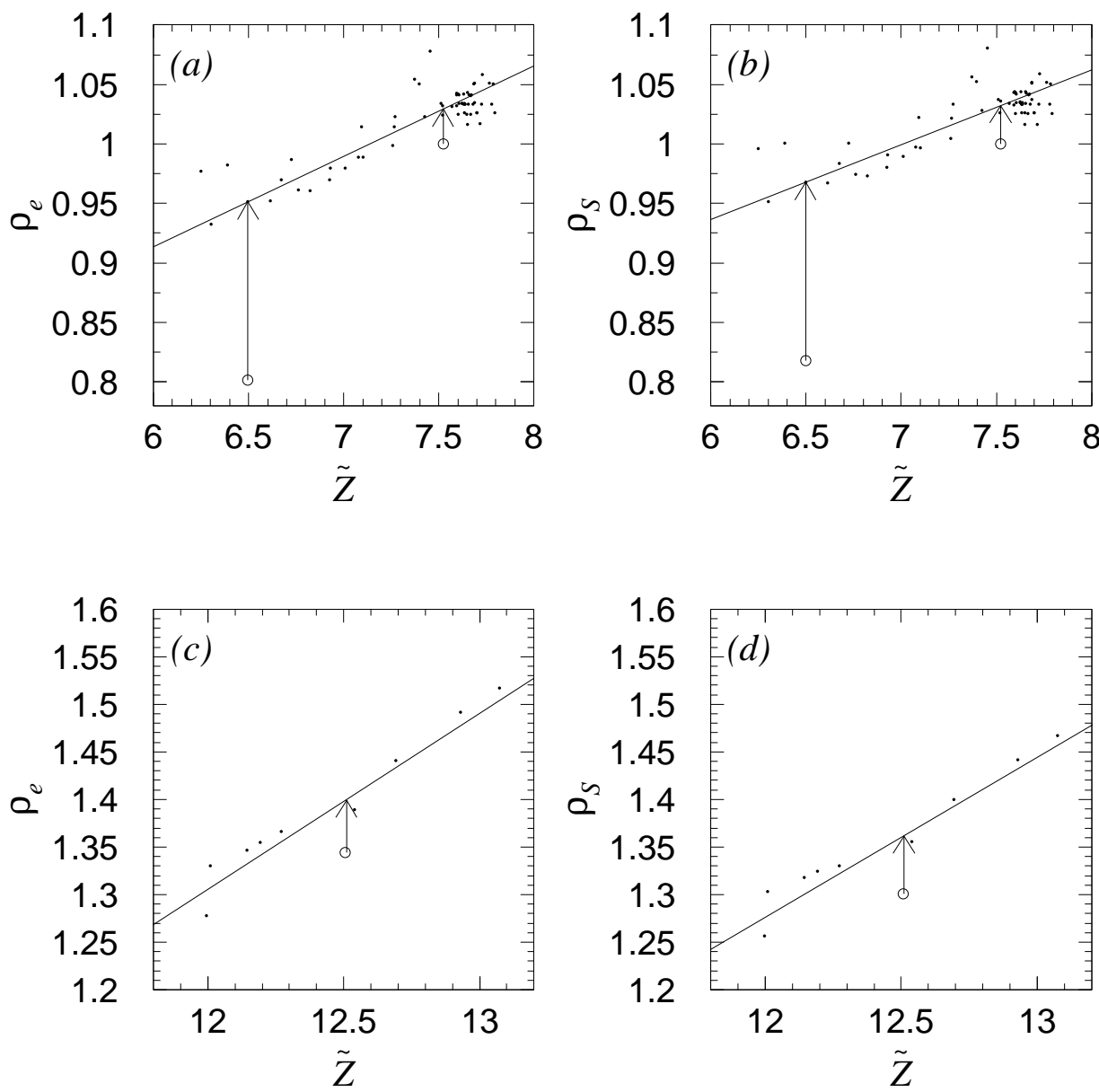

Figure 3. Correlation between effective atomic number and effective density; $(a)$ $\tilde{Z}-\rho_{\mathrm{e}}$ for soft tissues, (b) $\tilde{Z}-\rho_{\mathrm{S}}$ for soft tissues, (c) $\tilde{Z}-\rho_{\mathrm{e}}$ for bones, $(d) \tilde{Z}-\rho_{\mathrm{S}}$ for bones, where the lines are the results of linear fits, the circles indicate ethanol, water, and $40 \%-\mathrm{K}_{2} \mathrm{HPO}_{4}$ solution, and the arrows schematically represent the scaling corrections.

\subsection{The polybinary calibration procedure}

The polybinary calibration with the selected tissue substitutes can be carried out in a simple procedure described as follows. The tissue substitutes, which are water, air, ethanol, and $40 \%-\mathrm{K}_{2} \mathrm{HPO}_{4}$ solution, filled in small hermetic containers placed in a calibration phantom are CT-scanned in the same manner as in the tissuesubstitute calibration. The CT numbers for ethanol and $40 \%-\mathrm{K}_{2} \mathrm{HPO}_{4}$ solution are then converted to $\mu_{\mathrm{E}}$ and $\mu_{\mathrm{K}}$, respectively, using (4). The observed values of $\mu_{\mathrm{E}}$ and $\mu_{\mathrm{K}}$ uniquely determine the polyline for a given scanning condition, according to table 2 . 
Table 3. Observed radiological properties, such as mean $m_{\mathrm{CT}}$ and standard deviation $\sigma_{\mathrm{CT}}$ of the $\mathrm{CT}$ numbers and relative range shift in water $R(\mathrm{~mm})$, for the calibration materials and the liquid biological materials.

\begin{tabular}{llll}
\hline Material & $m_{\mathrm{CT}}$ & $\sigma_{\mathrm{CT}}$ & $R$ \\
\hline Air & -1019.6 & 4.5 & 129.74 \\
Water & 11.4 & 6.7 & 33.87 \\
Ethanol & -222.0 & 6.0 & 51.28 \\
\hline Milk & 42.9 & 6.2 & 31.36 \\
Olive oil & -122.6 & 5.9 & 39.76 \\
\hline
\end{tabular}

\section{Results}

\subsection{Carbon range verifications with liquid biological materials}

We tested the polybinary calibration by comparing measured relative stopping powers with predictions from measured CT numbers using sample biological materials, assuming that they were equivalent to body tissues. We first used liquid materials, milk for a blood substitute and olive oil for a body-fat substitute, so that there would be little measurement errors caused by heterogeneity.

The calibration materials (air, water, and ethanol) and the biological materials (milk and olive oil) were separately filled in 5-cm diameter plastic containers and were then CT-scanned altogether. Table 3 shows the results of the CT scan, where the standard deviations comparable to that of water imply that they were actually homogeneous. We measured the relative stopping powers of the materials with the clinical carbon-ion beams at facility HIMAC (Kanai et al 1999) in a setup similar to those described by Schaffner and Pedroni (1998) and Jäkel et al (2001). The observed range shifts in table 3 by the materials in the box of $96-\mathrm{mm}$ spacing resulted in relative stopping powers 0.818 for ethanol, 0.939 for olive oil, and 1.026 for milk. The polybinary calibration gives a polyline from the CT numbers for ethanol and water, which should be compared to measured data points for milk and olive oil, as shown in figure 4 . The deviations of the points from the polyline were $-0.7 \%$ for milk and $-0.1 \%$ for olive oil in relative stopping power, which are consistent with the ideal resolution estimated in section 2.1 .

\subsection{Carbon range verifications with fine-ground biological materials}

The polybinary calibration was further tested with HIMAC in a more realistic situation. In this test, we used fatty meat, lean meat, and mixture of lean meat (400 g), hydroxyapatite (100 g), and water (150 g), after fine grinding and mixing as uniformly as possible, where the hydroxyapatite-added meat was to simulate a mixture of bone and muscle.

A CT scan of a cylindrical water-tank phantom containing samples of air, water, ethanol, and $40 \%-\mathrm{K}_{2} \mathrm{HPO}_{4}$ solution was immediately followed by another CT scan of the fresh biological materials stuffed in the plastic boxes. The boxes had the same cross section of approximately $100 \mathrm{~mm} \times 100 \mathrm{~mm}$ and gaps of $96 \mathrm{~mm}$ for the fatty meat, $71 \mathrm{~mm}$ for the lean meat, and $46 \mathrm{~mm}$ for the hydroxyapatite-added meat. Table 4 shows the results of the $\mathrm{CT}$ scans. The noticeable increases in standard deviation for the lean meat and the hydroxyapatite-added meat may be mostly caused by air 


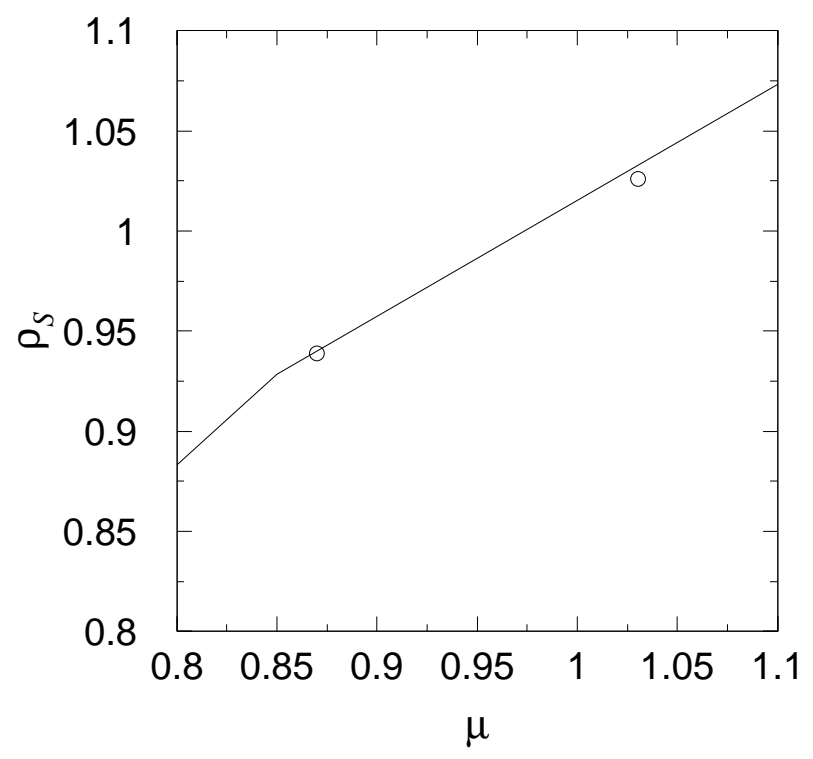

Figure 4. Test of the polybinary calibration for the liquid biological materials, where the solid line shows the calibrated $\mu-\rho_{S}$ relationship for body tissues and circle markers show the measured data points for olive oil at $\mu=0.87$ and milk at $\mu=1.03$.

contaminations that were actually visible in the scanned image.

We placed the biological materials on the beam path and measured the range shifts relative to water caused by the biological materials with the carbon beam that had been modified by a range-smearing filter of 2.5-mm Gaussian function (Schaffner et al 2000) to reduce the influences of the heterogeneity. Figure 5 shows the measured depth-dose curves and we measured the beam ranges by the $60 \%$-dose depths. Though the heterogeneity may have caused maximum $5 \%$ reduction in peak height, the influence to the range measurement was calculated to be negligible for the Bragg curve dominated by the Gaussian function, compared to $0.5 \mathrm{~mm}$ estimated for mechanical precision of the measurement system. The measured range shifts resulted in relative stopping powers 0.975 for the fatty meat, 1.015 for the lean meat, and 1.111 for the hydroxyapatite-added meat.

Figure 6 shows the polyline calibrated by the responses of ethanol and 40\%$\mathrm{K}_{2} \mathrm{HPO}_{4}$ solution (asterisks), the observed responses for the fine-ground biological materials (circles), and the ICRU body tissue responses (dots) predicted by the stoichiometric calibration with parameters $K^{\mathrm{ph}}=2.115 \times 10^{-5}, K^{\mathrm{coh}}=2.151 \times 10^{-3}$, and $\sigma^{\mathrm{KN}}=0.8858$, which were determined by solving equation (1) for the calibration materials. The fact such that polyline well agreed to the calculated body tissue responses concludes that the polyline calibration is equivalent to the stoichiometric calibration. The deviations of the measured biological materials from the polyline in relative stopping power were $+1.3 \%(+1.7 \sigma)$ for the fatty meat, $-0.9 \%(-0.9 \sigma)$ for the lean meat, and $-2.8 \%(-2.0 \sigma)$ for the hydroxyapatite-added meat, where $\sigma$ represents the standard error from the range measurement error of $0.5 \mathrm{~mm}$. Since the regions 
Table 4. Observed radiological properties, such as mean $m_{\mathrm{CT}}$ and the standard deviation $\sigma_{\mathrm{CT}}$ of the $\mathrm{CT}$ numbers, physical length $L(\mathrm{~mm})$, and range shift relative to water $R$ - $R_{\text {water }}(\mathrm{mm})$, for the calibration materials and the fine-ground biological materials.

\begin{tabular}{lllll}
\hline Material & $m_{\mathrm{CT}}$ & $\sigma_{\mathrm{CT}}$ & $L$ & $R$ - $R_{\text {water }}$ \\
\hline Air & -987.0 & 8.5 & & \\
Water & 18.5 & 7.1 & & \\
Ethanol & -210.2 & 8.6 & & \\
$40 \%-\mathrm{K}_{2} \mathrm{HPO}_{4}$ solution & 710.4 & 9.9 & & \\
\hline Fatty meat & -73.9 & 15.6 & 96 & -2.4 \\
Lean meat & 33.8 & 31.2 & 71 & 1.1 \\
Hydroxyapatite-added meat & 287.2 & 24.9 & 56 & 5.1 \\
\hline
\end{tabular}

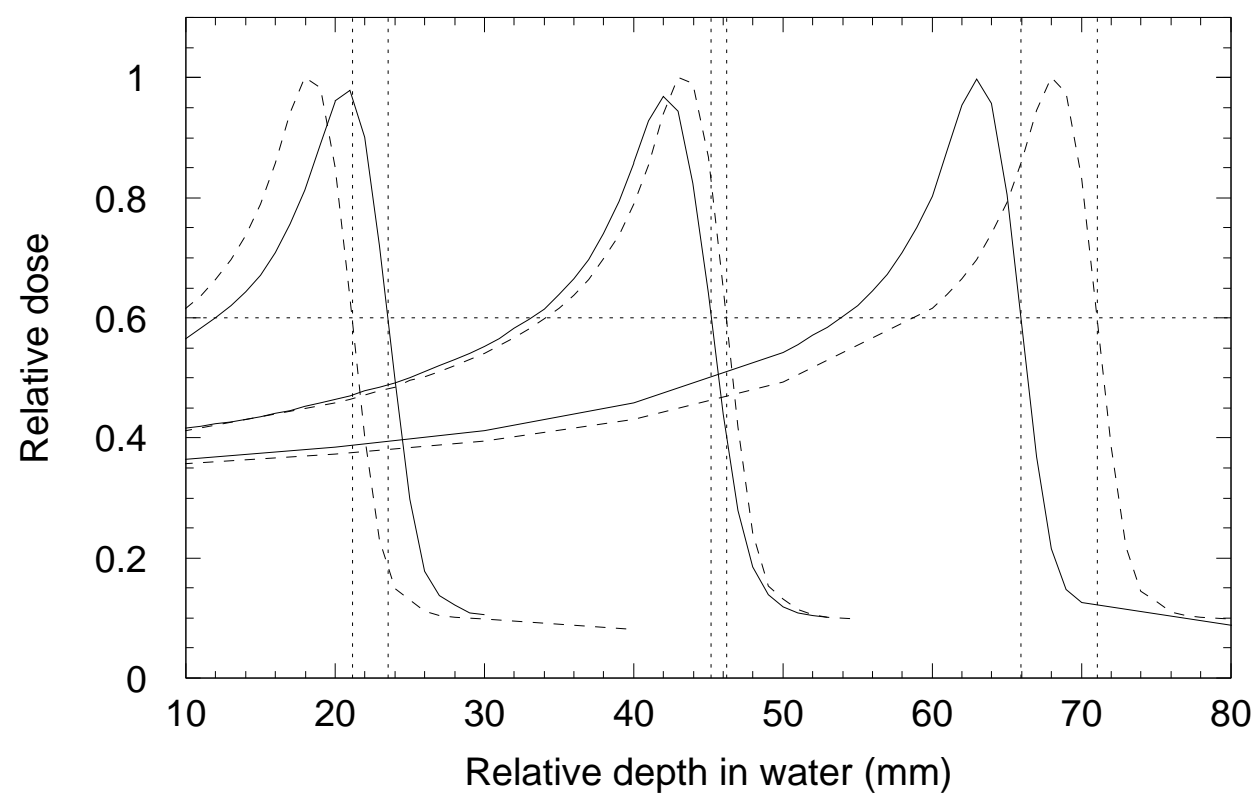

Figure 5. Gaussian-smeared Bragg peaks with absorbers 96-mm water (dashed line), 96-mm fatty meat (solid line), 71-mm lean meat (solid line), 71-mm water (dashed line), 46-mm hydroxyapatite-added meat (solid line), and 46-mm water (dashed line) in the ascending order of depth. The vertical dotted lines indicate the depths for the $60 \%$-dose level shown by the horizontal dotted line.

of interest for the CT measurements of the biological materials were not exactly the volumes relevant to the range measurements, the macroscopic heterogeneity due to air contamination could explain the observed deviations just as experimental errors.

\section{Discussion}

The physical models for the relevant radiations, the ICRU body tissue data, and use of a conventional CT scanner are the error sources common to both the stoichiometric 


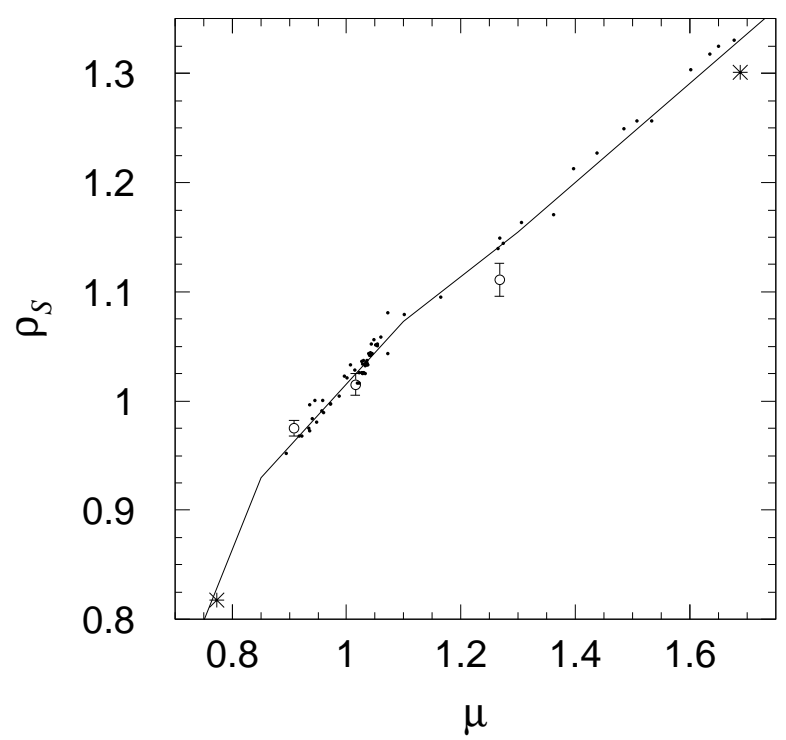

Figure 6. Test of the polybinary calibration for fine-ground biological materials, where the solid line shows the $\mu-\rho_{S}$ relationship calibrated for body tissues, the circle markers with error bars show the observed responses for the fatty meat, the lean meat, and the hydroxyapatite-added meat, the asterisks show the responses of ethanol and $40 \%-\mathrm{K}_{2} \mathrm{HPO}_{4}$ solution, and the dots show the ICRU body tissue responses predicted by the stoichiometric calibration.

and polybinary calibrations. Schneider et al (1996) and Schaffner and Pedroni (1998) already discussed most of them in detail, except for the accuracy of equation (1). White (1977) made energy and material dependent analysis on the approximation and found some variations of exponents $m$ and $n$ for the effective atomic numbers. Figure 7 shows the effective atomic numbers for the substitutes (ethanol, water, and $40 \%-\mathrm{K}_{2} \mathrm{HPO}_{4}$ solution) and the nearest tissues (adult adipose tissue, GI tract, and skeleton humerus, respectively). Water and ethanol showed quite good similarity to the tissues, which assures that the polybinary calibration will be insensitive to the exponent variations. Behaviors of $40 \%-\mathrm{K}_{2} \mathrm{HPO}_{4}$ solution and the nearest bone (skeleton humerus) are substantially different and equation (1) predicts $\mu$ uncertainty of $1 \%$ level. However, the $1 \%$ error for bones should be negligible to effective depth, considering that the bone content is only $7 \%$ in Reference Man by ICRP (1975).

Schaffner and Pedroni (1998) estimated the accuracy of the stoichiometric calibration to $1 \%$ in effective density and overall errors to $1.1 \%$ for soft tissues and $1.8 \%$ for bones including beam hardening artifacts in typical planning CT scans, which should be also applicable to the polybinary calibration. Since the integral calculation will reduce the relative error, the depth error would be better than $1 \%$ in usual clinical situations with small bone content.

The polybinary calibration requires only a CT scan of a fat substitute (ethanol) and a bone substitute $\left(40 \%-\mathrm{K}_{2} \mathrm{HPO}_{4}\right)$ besides water and air. In addition, the calculations to deduce the relationship only include simple arithmetic operations 


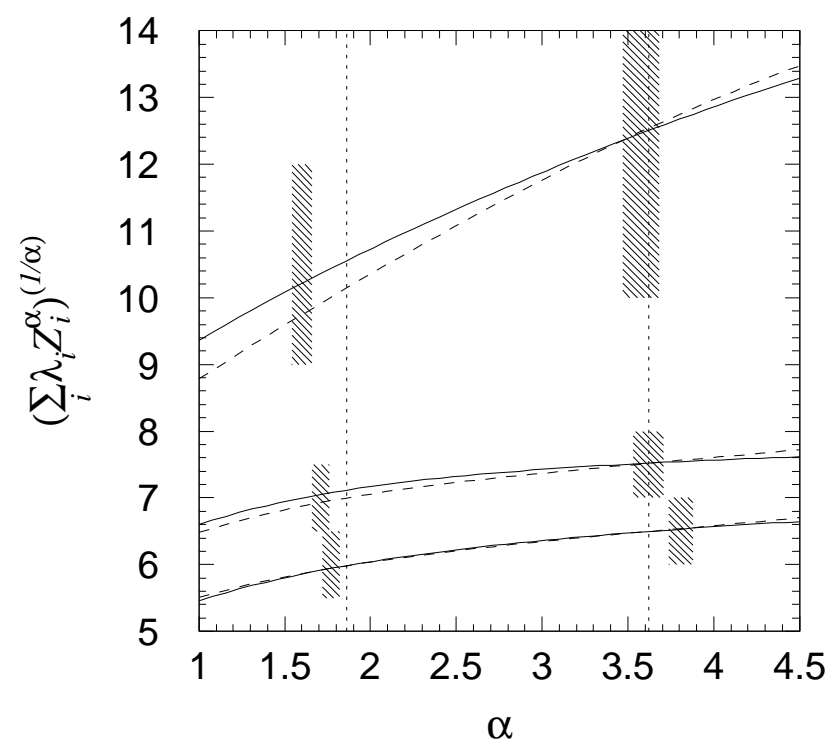

Figure 7. Exponent $(\alpha)$ dependences of the effective atomic numbers for the tissue substitutes (ethanol, water, and $40 \%-\mathrm{K}_{2} \mathrm{HPO}_{4}$ solution) in solid lines, and those for the nearest ICRU tissues (adult adipose tissue, GI tract, and skeleton humerus, respectively) in dashed lines. The vertical dotted lines indicate exponents $m$-1 (3.62) and $n$-1 (1.86) while the hatched regions indicate their variations for $40-140 \mathrm{keV}$ photons reported by White (1977).

with several definite constants. Simplicity and specificity should be mandatory for a standard that has to be routinely practiced by the relevant facilities with the same quality. Furthermore, the simplicity will possibly enable the self-calibration of planning CT images if the patient is scanned with the calibration materials appropriately placed in the scanning field.

\section{Conclusions}

We have established an extremely simple and very accurate method, the polybinary calibration, to determine the relationship between CT number and the effective density for radiotherapy. As a result of the reasonable approximations to the stoichiometric calibration, the polybinary calibration eliminated the complicated calculations from the calibration procedure while achieving the equivalent calibration accuracy better than $1 \%$ for depth in usual clinical situations. We propose this method as a standard for radiotherapy treatment planning, in particular, with heavy charged particles.

\section{Acknowledgments}

This work was motivated by a discussion on quality assurance of heavy charged particle radiotherapy with relevant facilities in Japan including University of Tsukuba, National Cancer Center (Kashiwa), Hyogo Ion Beam Medical Center, The Wakasa 
Wan Energy Research Center, and Shizuoka Cancer Center. We thank them for the opportunity and their cooperation. We are also indebted to Dr Uwe Schneider and Dr Eike Rietzel for helpful advice in the beginning of this work.

We thank researchers and technical staff at NIRS, in particular, Dr Masahiro Endo, Dr Eiichi Takada, Dr Masami Torikoshi, Dr Akifumi Fukumura, Dr Ken Yusa, and Dr Takanori Tsunoo for valuable discussions and Kobayashi, Uno, Ikeda, Nishimura, Hasegawa, Kohno, and Kusano for technical support.

\section{References}

Chen G T Y, Singh R P, Castro J R, Lyman J T and Quivey J M 1979 Treatment planning for heavy ion radiotherapy Int. J. Radiat. Oncol. Biol. Phys. 5 1809-1819

Constantinou C, Harrington J C and Dewerd L A 1992 An electron density calibration phantom for CT-based treatment planning computers Med. Phys. 19 325-327

ICRP 1975 Reference man: anatomical, physiological and metabolic characteristics ICRP Publication 23 (Oxford: Pergamon Press)

ICRU 1984 Stopping powers for electrons and positrons ICRU Report 37 (Bethesda, MD: ICRU)

1989 Tissue substitutes in radiation dosimetry and measurement ICRU Report 44 (Bethesda, MD: ICRU)

1992 Photon, electron, proton and neutron interaction data for body tissues ICRU Report 46 (Bethesda, MD: ICRU)

1993 Stopping powers and ranges for protons and alpha particles ICRU Report 49 (Bethesda, MD: ICRU)

IEC 1994 Evaluation and routine testing in medical imaging departments - Part 2-6: Constancy tests - X-ray equipment for computed tomography IEC Standard 61223-2-6 (Geneva: IEC)

Jackson D F and Hawkes D J 1981 X-ray attenuation coefficients of elements and mixtures Phys. Rep. 70 169-233

Jäkel O, Jacob C, Schardt D, Karger C P and Hartmann G H 2001 Relation between carbon ion ranges and x-ray CT numbers Med. Phys. 28 701-703

Kanai T, Endo M, Minohara S, Miyahara N, Koyama-Ito H, Tomura H, Matsufuji N, Futami Y, Fukumura A, Hiraoka T, Furusawa Y, Ando K, Suzuki M, Soga F and Kawachi K 1999 Biophysical characteristics of HIMAC clinical irradiation system for heavy-ion radiation therapy Int. J. Radiat. Oncol. Biol. Phys. 44 201-210

Kutcher G J, Coia L, Gillin M, Hanson W F, Leibel S, Morton R J, Palta J R, Purdy J A, Reinstein L E, Svensson G K, Weller M and Wingfield L 1993 Comprehensive QA for radiation oncology: Report of AAPM Radiation Therapy Committee Task Group 40 Med. Phys. 21 581-618

Matsufuji N, Tomura H, Futami Y, Yamashita H, Higashi A, Minohara S, Endo M and Kanai T 1998 Relationship between CT number and electron density, scatter angle and nuclear reaction for hadron-therapy treatment planning Phys. Med. Biol. 43 3261-3275

Rietzel E, Geiß O, Schardt D, Voss B, Krämer M and Haberer T 2000 Correlation between CT number and water-equivalent thickness for heavy charged particles Proc. 7th Workshop on Heavy Charged Particles in Biology and Medicine (Darmstadt, Germany)

Rutherford R A, Pullan B R and Isherwood I 1976 Measurement of effective atomic number and electron density using an EMI scanner Neuroradiology 11 15-21

Schaffner B and Pedroni E 1998 The precision of proton range calculations in proton radiotherapy treatment planning: experimental verification of the relation between $\mathrm{CT}-\mathrm{HU}$ and proton stopping power Phys. Med. Biol. 43 1579-1592

Schaffner B, Kanai T, Futami Y and Shimbo M 2000 Ridge filter design and optimization for the broad-beam three-dimensional irradiation system for heavy-ion radiotherapy Med. Phys. $\mathbf{2 7}$ 716-724

Schneider U, Pedroni E and Lomax A 1996 The calibration of CT Hounsfield units for radiotherapy treatment planning Phys. Med. Biol. 41 111-124

White D R 1977 An analysis of the Z-dependence of photon and electron interactions Phys. Med. Biol. 22 219-228 